\title{
Experimental determination of invasive fitness in Caenorhabditis elegans
}

\author{
Ivo M. Chelo \\ Instituto Gulbenkian de Ciência, Apartado 14, P-2780-156 Oeiras, Portugal \\ imchelo@igc.gulbenkian.pt
}

Phone number: +351214464694

Fax number: +351214407970

\begin{abstract}
SUMMARY:
Estimation of fitness is a key step in experimental evolution studies. However, no established methods currently exist to specifically estimate how successful new alleles are in invading populations. The main reason is that most assays do not accurately reflect the randomness associated with the first stages of the invasion, when invaders are rare and extinctions are frequent. In this protocol, I describe how such experiments can be done in an effective way. Using the nematode model, Caenorhabditis elegans, a large number of invasion experiments are set up, whereby invading individuals carrying a visual marker are introduced into populations at very low numbers. The number of invaders counted in consecutive generations, together with the number of extinctions, is then used in the context of individual-based computer simulations to provide likelihood estimates for fitness. This protocol can take up to five generations of experimental invasions and a few hours of computer processing.
\end{abstract}

Keywords : Fitness; Invasion; Extinction; Simulation; Caenorhabditis elegans.

Suggested article: Chelo, I. M., Nédli, J., Gordo. I. \& Teotónio, H. An experimental test on the probability of extinction of new genetic variants. Nat. Commun. 4, 2417 (2013). doi:10.1038/ncomms3417 


\section{INTRODUCTION}

Evolution is highly dependent on how different alleles or genotypes influence the individual's offspring contribution to the following generations, that is, their 'fitness'. In fact, the fitness effects of alleles, together with the frequency at which they appear in populations, determine how fast adaptation can occur. Unfortunately, knowing what evolutionary rates to expect is not easy. First, it can be experimentally challenging to determine if and how different alleles or genotypes affect fitness, given that measurable phenotypes (fitness components) may not translate well into total lifetime fitness or may not be comparable among different species ${ }^{1}$. Second, to get any prediction about evolutionary dynamics, one has to estimate the relative fitness of genotypes, in other words, one has to be able to determine the fitness of genotypes with respect to other genotypes present in the populations ${ }^{2}$. Finally, even when the relative fitness of a new allele is known, its fixation in the population is contingent on it not being lost by drift while it is still rare $\mathrm{r}^{3,4}$. Drift is an important determinant of the probability of a genotype to invade and become established in a population characterized by other genotypes ${ }^{5,6}$ and, ultimately, a key outcome to evaluate in order to understand the role of fitness in evolution ${ }^{7}$.

Herein, I describe a method whereby fitness is estimated on the basis of the outcome of invasion experiments, which are conducted by introducing a low number of individuals harboring a given genotype into a resident population of individuals with a different genotype. For that purpose, two different isogenic lines of the nematode Caenorhabditis elegans are used in which the invader carries a visible marker, obtained by expressing a green fluorescent protein (GFP). Frequency trajectories are obtained, by counting GFP individuals in a population of known size for a small number of generations, which are then used to infer a relative fitness measure by comparison with computer simulations of the invasions. The present protocol is conceptually simple and can be used to describe fitness variation in many other organisms.

\section{Development of the protocol}

This protocol was developed to experimentally demonstrate the role of genetic drift in the extinction probability of new genetic variants ${ }^{8}$, a parameter which affects fixation probabilities and, ultimately, the rate of evolution. The importance of sampling effects during invasions of beneficial alleles was deduced in the early developments of population genetics ${ }^{4}$. Briefly, random fluctuations of allele frequencies, which characterize genetic drift, occur because populations are finite and the genetic pool of the following generations is obtained by a random sampling of alleles. After the appearance of a new allele by mutation, these fluctuations often result in extinction of the allele, even if it confers a fitness advantage ${ }^{4,9}$. For this reason, an experimental setup to reflect these 'chance dynamics' must enable the experimenter to control precisely the initial number of invaders 
that harbor the new alleles. Furthermore, since drift effects are reflected in the variance of frequency trajectories, a large number of replicate experiments have to be employed, which will also provide the means to measure extinction rates.

C. elegans was chosen to experimentally model the invasion dynamics of beneficial alleles in populations mainly because, by following standard maintenance procedures ${ }^{10,11}$, it can be made to conform to the simplest scenario of the Wright-Fisher model of allele frequency change, where all individuals reproduce at the same time (generations are discrete), the offspring replaces progenitors in the population (generations are non-overlapping), and populations sizes are high and constant ${ }^{2}$. Furthermore, C. elegans isogenic populations (lines), in which all individual worms have the same genotype, can be obtained by recurrent self-fertilization of hermaphrodites. Generation of these isogenic populations will ensure that their relative fitness is constant throughout the experiments, as long as there is no time for new mutations to appear and rise to substantial frequency. In the study on which the present protocol is based $^{8}$, the same genetically variable population, which had previously adapted to laboratory conditions, was used to derive two isogenic lines. ${ }^{11,12}$. In the line to be used as the invader, an extra step was added before the derivation of individuals by selffertilization, which consisted in the introgression of a genetic construct into the ancestral population that leads to the constitutive expression of a $\mathrm{GFP}^{8,13}$. This genetic modification was implemented to enable the experimenter to easily discriminate between individuals belonging to the two different lines. Finally, since worms can be individually picked and transferred between populations it is possible to establish invasions with a predetermined low number of individuals, an ability that is crucial to test for the effects of different levels of genetic drift ${ }^{8}$.

\section{Experimental design}

The design employed in this protocol is schematized in Figure 1. It encompasses an experimental stage (Figure 1a), where the goal is to obtain frequency trajectories from observed invader counts, and computer simulations (Figure 1b) to generate simulated frequency trajectories from different selection coefficients, and thus find the most likely fitness value for the invading genotypes.

Throughout the protocol, general maintenance procedures follow standard practices in $C$. elegans research ${ }^{10,11}$. The laboratory protocol begins with a preliminary population growth stage, in which the competing C. elegans lines are revived from stocks kept at $-80{ }^{\circ} \mathrm{C}$ and maintained for two generations in common laboratory conditions to synchronize them and to remove possible effects of specific interactions with storage conditions and trans-generational effects (parental effects). The following invasion assay starts with the transfer of a low number $(n)$ of individuals carrying the invading genotypes into a population carrying the 'resident' genotypes. Since the results will consist 
of counts of invading individuals through time, it is generally preferable that the marked, GFPexpressing in this case, individuals are used as invaders. In the case in which the population size is not easily determined beforehand, and both types of individuals have to be counted simultaneously, the distinction is irrelevant.

In practice, the decision of whether a marked or unmarked genotype is to be used as an invader is a pragmatic one. Even though GFP expression is used in this protocol as a functional way of distinguishing invading individuals from the resident population, any kind of visual marker can be used for this purpose, as long as it is stable. In any case, caution should be used in the interpretation of results, since the marker itself is likely to affect fitness. Moreover, it is important to make sure that the mixing of invaders into the resident population is done at the same stage of the life cycle at which the counting will take place, so that changes in frequencies will be based on complete generations. If this is not possible, one can use frequency estimates performed one or two generations after the start of invasion as initial counts. The disadvantage of this approach is that it will prevent a complete control of the initial invader numbers.

After the invasion has been set up, independent replicates are maintained for several generations while GFP-expressing individuals are counted in each replicate, by observation under a microscope equipped with fluorescent light and GFP filter. Scoring is always conducted at the same life stage, and it can take place in every generation or only in a subset of generations. Even though the longer the experiment is run the more data can be retrieved, the number of generations should be kept low. This strategy is important to reduce the number of mutations appearing during the experiment and to avoid that any mutation reaches a high frequency in most replicates. Such high frequency across replicates can occur when the selection coefficients of the invaders is high; the scenario in which the invading population reaches high frequency across several replicates should be avoided because when it occurs the invaders' growth dynamics is no longer reflected by a branching process ${ }^{3,4}$, in which individuals can be modeled as either dying or splitting into a random number of offspring, which behave identically, but independently, of their progenitors.

In the second part of the protocol, invasion simulations and estimation of selection coefficients are performed to infer possible frequency trajectories, using the same generations that were experimentally recorded, and starting from different fitness values. The use of selection coefficients (s) to measure relative fitness was adopted since the latter can be interpreted as the difference between the long-run (logarithmic) growth rates of the invader and resident population ${ }^{14}$. Describing relative fitness in this manner, assumes that the invaders have a $\boldsymbol{w}=\mathbf{1}+\boldsymbol{s}$ fitness advantage over the resident genotypes. These simulations take advantage of the experimental constraints enforced on the procedure, such as constant population size, discrete generation cycles, and 
sampling from a finite population. Likelihood (Lk) estimates of the observed data under different selection coefficients are obtained by recording the frequency at which they appear in the simulations. Finally, to obtain a maximum Lk estimate together with a confidence interval, a local fit with a quadratic curve is performed, using only the selection coefficients with the higher Lk.

A script file in $\mathrm{R}_{\text {language }}{ }^{15}$ is provided as the Supplementary Data, together with an example table of counts (Supplementary Table 1), with the full implementation of the invasion algorithm and subsequent analysis. This script was implemented with $\mathrm{R}$ version 3.0.5 and it can be run by copying and pasting the command lines into a graphical user interface (GUI) in windows or directly into a shell executing $\mathrm{R}$ in a Linux system. Note that it is expected that the Supplementary Table 1 file is in the same environment where $\mathrm{R}$ is being run.

\section{Comparison with other methods}

Many approaches to estimate fitness are well known in evolutionary biology literature ${ }^{1}$, and one method that has been used extensively involves competition experiments between individuals with different genotypes ${ }^{8,16,17}$. In this approach, relative fitness is estimated from the change in frequencies through time (through the generations) of the different genotypes present in a mixed population ${ }^{2,18}$. This kind of experiment has the advantage that the comparison of genotype fitness rely on total lifetime measures rather than on fitness components, such as fecundity or viability, and thus present a clear connection to the measure of possible evolutionary success, fitness ${ }^{1}$. Nevertheless, fitness estimates obtained in this manner do not necessarily give a measure of invasion ability, since factors such as frequency- or density- dependence effects may be present, thus these estimates do not reflect the intrinsic growth rate of a given genotype ${ }^{1,9,19}$. To estimate the intrinsic growth rate, the invading genotype must be rare, so that interactions between individuals with that genotype are negligible, and the overall dynamics of the population, which are determined by the resident genotypes, do not change $\mathrm{e}^{20}$. Under those these circumstances the selection coefficient of the invader genotype reflects its long-term per capita growth rate relative to resident genotype and determine the probability of a successful invasion ${ }^{7}$.

From an experimental point of view, the need to specifically address invasive fitness is obvious, as shown by Gifford and colleagues ${ }^{6}$, who used strains of the fungus Aspergillus nidulans to characterize the dependence of extinction on selection strength. In their case, however, the initial number of invading individuals in each replicate could not be known with certainty, but had instead to be included as a variable in the analysis.

\section{Limitations}

Most of the limitations of this protocol also apply to other methodologies that measure 
competitive fitness.

The first such limitation concerns the need to use a visible marker (GFP expression in the present protocol) to tag one of the strains or lines. In fact, the effect of the genotype of one of the competitors will be indistinguishable from the effect of the marker. One possible solution to this confounding effect is to use the marked genotype as the resident genotype, since there is no a priori reason for the invading genotype to be the unmarked one. When implementing this approach, detecting the number of invaders may require more time, and this timing requirement to an extent is likely to be species- and marker-specific. Then, comparison of the invasion ability between different genotypes can be carried out by conducting invasion experiments against the same, marked, reference genotype, an approach that only works if fitness is transitive. An alternative solution, though a more laborious one, is to compare the results from duplicate experiments were the marker is introduced either in the invading or in the resident genotype. The invasion ability could then be obtained from the mean of the fitness estimates obtained in the two experiments. However, note that changing the roles between the competing genotypes does not necessarily lead to equivalent relative fitness estimation. In this protocol, invasion ability of a genotype comes from the growth rate of the invading genotype in a population whose growth dynamics are determined by the resident genotype's. Depending on how individuals of each genotype behave when rare or frequent, or depending on how they interact with individuals of the other genotype, it is possible that the best genotype at invading is the worst at resisting invasion ${ }^{21}$.

A second limitation, specific to this protocol, comes from the need to know exactly the number of invaders that are introduced. This experimental goal is only achievable by manipulating single individuals which, in general, may limit the number of species that can be used. Furthermore, in situations where the handling of individuals can be harmful, such as when working with $C$. elegans, a bias may be introduced if invading and resident individuals are treated differently. In fact, in this protocol, the introduction of $C$. elegans invaders is performed by single individual picking, whereas resident genotypes are transferred in liquid buffer as L1-staged larvae. Beyond the obvious precautions that are taken during the transfer of invading individuals, some procedures can help reduce or remove this problem, though they are not without potential pitfalls themselves. One precaution, and the one taken in this protocol, is based on the consideration that the detrimental effect caused by manipulation of individuals carrying the invading genotype should mainly affect frequency changes in the first generation. Using data from later generations is expected to partially mitigate the above mentioned bias, as frequency changes during those generations should be unaffected by this initial problem. Another precaution consists in forcing invading and resident individuals to switch roles, as mentioned above. Yet another option is to apply the same procedure to both sets of individuals. However, if the procedure is time-consuming it may be impractical to 
apply it to thousands of individuals of the resident population. For C. elegans, one interesting solution would be to use microfluidic devices, which can efficiently manipulate single as well as thousands of individuals ${ }^{22}$.

In this protocol, the duration of the experiment is also a factor that needs to be planned carefully. Performing the experiment over too many generations will increase the risk of new mutations but also, by definition, invasive fitness can only be determined in the first few generations after the invasion. At the same time, given that more generations increase exponentially the number of possible trajectories that need to be taken into account, a much larger simulation effort would have to be made. Otherwise, the sequence of counts observed in some replicates would never be retrieved in the simulations and this would lead to problems in the estimation procedure (see Supplementary Figure 1). On the other hand, too few generations are likely to result in low power to estimate fitness, especially given that in many replicates the invading genotype will become extinct soon after the start of the experiment. The solution to this problem is to increase the number of invasion replicates. Yet, the number of experimental replicates that need to be used is a question that ultimately depends on factors such as the selection coefficient and the amount of drift in each experimental system, which are obviously unknown parameters in most cases. Figure 2 provides some guidelines on how the number of invasion replicates influence the estimation procedure. First, it is interesting to see that throughout the range of selection coefficients used, 30 replicate experiments are enough to provide accurate estimates (Fig. 2a). Lower numbers of replicates (10) results in a negative bias for selection coefficients below 0.1 . This bias is likely a result of the undue weight of trajectories with early extinctions, which are always better explained with smaller, even negative, selection coefficients. Note that this type of trajectory is the most frequent when invasions start with only a few individuals and as long as selection coefficients are not too high. The power of the estimation procedure, here defined as the probability of the true selection coefficient being in the estimated $-2 \times \operatorname{lnLikelihood~(lnLk)~interval,~is~generally~high~}$ (above 0.9). Surprisingly though, an increase in the number of experimental replicates is not necessarily associated with an increase in power. This lack of association is probably caused by the fact that the most significant effect of increasing the number of replicates is to increase the precision of the estimation, measured as the size of the confidence interval, as seen in Fig.2c. Note that, all else being equal, this increase must come with a cost in 'power', which can explain why the use of more experimental replicates does not necessarily results in more power to detect the 'true' selection coefficient, as seen in Fig.2b. 


\section{MATERIALS}

\section{REAGENTS}

- Nematode growth medium (NGM lite) (Europa Bioproducts, cat. no. UBN1005)

- Sodium hypochlorite $(\mathrm{NaOCl})$ solution, 12\% (wt/vol of active chlorine) (ROTH, cat. no. 9062.3) ! CAUTION Hypochlorite is corrosive and dangerous for the environment. Wear a lab coat and protective gloves when handling it. This chemical should be kept away from acids.

- Potassium hydroxide (KOH, Sigma-Aldrich, cat. no. 1310-58-3) ! CAUTION Potassium hydroxide is corrosive and toxic. Wear a lab coat and protective gloves when handling it.

- Ampicillin sodium salt (Sigma-Aldrich, cat. no. 69-52-3)

- Potassium dihydrogen phosphate $\left(\mathrm{KH}_{2} \mathrm{PO}_{4}, \mathrm{ROTH}\right.$, cat. no. P018.2)

- Sodium hydrogen phosphate $\left(\mathrm{Na}_{2} \mathrm{HPO}_{4}, \mathrm{ROTH}\right.$, cat. no. T876.2)

- Sodium chloride (NaCl, ROTH, cat. no. P029.3)

- Magnesium sulfate heptahydrate $\left(\mathrm{MgSO}_{4} \cdot 7 \mathrm{H}_{2} \mathrm{O}, \mathrm{ROTH}\right.$, cat. no. 0261.2)

- Double distilled water $\left(\mathrm{ddH}_{2} \mathrm{O}\right)$

- Escherichia coli HT115 (with L4440 plasmid, ampicillin resistant)

- Caenorhabditis elegans PD4251 strain (ccIs4251 I; dpy-20(e1282) IV), harboring the transgenic array ccls4251 (myo3::GFP) ${ }^{13}$, can be obtained from the Caenorhabditis Genetics Center (CGC).

- Caenorhabditis elegans experimental strains. Strains used in the initial implementation of the $\operatorname{protocol}^{8}$ are available upon request.

\section{EQUIPMENT}

- 92 mm x 16 mm Petri dishes (Sarstedt, cat. no. 82.1473)

- 15-ml conical tubes (TPP, cat. no. 91015) 
- Pasteur pipettes (VWR, cat. no. 612-1701)

- Serological pipettes $5 \mathrm{ml}$ (Sarsted, cat. no. 86.1253)

- Pipette filler (Fisher Scientific, cat. no. 888B8)

- Rubber bulbs (Fisher Scientific, cat. no. 5113B)

- Stereomicroscope equipped with fluorescent light, off-axis illumination (Zeiss SteREO Lumar.V12) and remote controller (HIP - human interface panel)

- Dissecting stereomicroscope (Nikon SMZ1500)

- Floor centrifuge (5810, Eppendorf)

- Swing-bucket rotor (Eppendorf, cat.no. A-4-81)

- Incubator with controlled humidity, (Aralab, ClimaPlus 400)

- Incubation shaker (Lab Companion, SK-600)

- Timer (Fisher Scientific, cat. no. S02316)

- Platinum wire (0.20 mm diameter, Refining Systems, Inc.)

- Workstation running $2.93 \mathrm{GHz}$ Intel ${ }^{(\mathrm{R})}$ Core $^{(\mathrm{TM})}$ i7 CPU with 16 Gb RAM.

\section{REAGENT SETUP}

Nematode growth medium plates. Dissolve $29.0 \mathrm{~g}$ of NGM lite in 11 of $\mathrm{ddH}_{2} \mathrm{O}$ (double distilled water) by stirring gently with heating until completely solubilized. Sterilize by autoclaving and let it cool down to around $50^{\circ} \mathrm{C}$. Add ampicillin to a final concentration of $50 \mathrm{mg} / \mathrm{ml}$ and pour $27 \mathrm{ml}$ of the resulting solution into each $92 \mathrm{~mm}$ x $16 \mathrm{~mm}$ Petri dish. These dishes can be stored safely at $4{ }^{\circ} \mathrm{C}$ for up to 2 months.

Seeding NGM plates. Pipette $100 \mu \mathrm{l}$ of of E. coli (HT115 strain), grown overnight at $37^{\circ} \mathrm{C}$ with constant shaking at $180 \mathrm{rpm}$, into a Petri dish and spread it with the help of glass beads by applying vigorous shaking. Incubate for $24 \mathrm{~h}$ at $37^{\circ} \mathrm{C}$ until bacterial growth results in a fully confluent lawn. Plates can be stored at $4{ }^{\circ} \mathrm{C}$ for up to 3 weeks.

$\mathrm{MgSO}_{4}$ solution, 1 M. Dissolve $246.48 \mathrm{~g}$ of $\mathrm{KOH}$ in 11 of $\mathrm{ddH}_{2} \mathrm{O}$ water. Sterilize by autoclaving and store it at room temperature $\left(15-25^{\circ} \mathrm{C}\right)$ for up to 12 months.

M9 buffer. Dissolve $3 \mathrm{~g}$ of $\mathrm{KH}_{2} \mathrm{PO}_{4}, 6 \mathrm{~g}$ of $\mathrm{Na}_{2} \mathrm{HPO}_{4}, 5 \mathrm{~g}$ of $\mathrm{NaCl}$ and $1 \mathrm{ml}$ of $\mathrm{MgSO}_{4}(1 \mathrm{M})$ in 11 of $\mathrm{ddH}_{2} \mathrm{O}$. Sterilize by autoclaving and store it at room temperature for up to 12 months.

KOH solution, 1 M. Dissolve $56.11 \mathrm{~g}$ of $\mathrm{KOH}$ in 11 of $\mathrm{ddH}_{2} \mathrm{O}$ water. Solution should be stored in 
plastic bottles. In these conditions the solution can be stored at room temperature indefinitely.

KOH:NaOCl solution. Add $20 \mathrm{ml}$ of $\mathrm{KOH} 1 \mathrm{M}$ and $50 \mathrm{ml}$ of $\mathrm{NaOCl} 12 \%$ (wt/vol of active chlorine) to $500 \mathrm{ml}$ of $\mathrm{ddH}_{2} \mathrm{O}$, in a glass container. Wrap the container in aluminum foil to protect the solution from light and store at $4{ }^{\circ} \mathrm{C}$ for up to 1 week.

Generating GFP expressing $C$. elegans populations by introgression. Introgression of the dominant GFP expression marker can be obtained by repeated backcrossing of the GFP positive individuals into the receiver populations (or isogenic lines). Cross 7 young adult or L4 males of the PD4251 GFP-expressing strain, by placing them with 24 hermaphrodites of the receiving population (or isogenic line), of the same developmental stage. From the resulting offspring (F1), select again 7 young adult or L4 males, which show GFP expression, to cross them again with 24 hermaphrodites of the receiving population. Repeat each crossing scheme for 10 introgression cycles to reduce the proportion of initial genetic background where the GFP locus was present, to expected levels below $0.1 \%$. In each cycle, that proportion is reduced by half, assuming that one crossing-over event occurs per chromosome and per meiosis. Finally, separate single hermaphrodites into individual plates and let them reproduce by self-fertilization. In each, check that all the progeny expresses GFP, as a means to identify those families where the GFP allele is in homozygosity. Use only those families to build a single population of GFP expressing individuals.

\section{EQUIPMENT SETUP}

Lighting conditions for counting. Set up the illumination (transmitted light) on the stereomicroscope to a comfortable minimum, so that it is possible to simultaneously use fluorescent light and regular light to identify GFP expression in the worms.

Worm picker. Cut a $2.5-3-\mathrm{cm}$ piece of platinum wire and use a flame to weld it into the tip of a Pasteur pipette. Flatten the extremity of the wire with a hammer.

\section{PROCEDURE}

\section{Preliminary population growth}

Timing 12 d (3 generations)

1 For each C. elegans line, take a frozen vial from a stock kept at $-80{ }^{\circ} \mathrm{C}$ and let it thaw to room temperature. 
2 Make sure the content of the vial is well mixed by vortexing the vial and then pour its content onto a NGM plate seeded with bacteria.

3 Let the worms develop for $72-80 \mathrm{~h}$, at $20^{\circ} \mathrm{C}$ and $80 \%$ relative humidity.

CRITICAL STEP The developmental time reported here is aimed at maximizing the number of progeny that can be recovered in the initial revival from frozen stocks. In practice, the optimal time is when most individuals have already started laying eggs, which can be easily seen on the agar with the stereoscope at 20x magnification.

$4 \quad$ Prepare $15-\mathrm{ml}$ tubes, one for each line, and add to each tube $10 \mathrm{ml}$ of M9.

5 Wash the worms off the plates with M9 buffer and transfer them to new 15-ml tubes (empty). Use for this purpose the 5-ml pipettes by pouring the M9 directly onto the plate and aspirating it afterwards. Repeat the procedure until the agar looks devoid of worms and remaining debris (it usually it takes 3-5 cycles). Be careful not to disturb the agar while pouring and aspirating the M9.

6 Centrifuge the $15-\mathrm{ml}$ tubes from step 5 at $650 \mathrm{~g}$ for $1 \mathrm{~min}$, at room temperature, and discard the amount of supernatant M9 necessary to keep only $5 \mathrm{ml}$ of supernatant in the tubes.

7 Take the $\mathrm{KOH}: \mathrm{NaOCl}$ solution from the fridge and immediately pour $5 \mathrm{ml}$ of this solution into the $15-\mathrm{ml}$ tube containing the suspension with the washed worms. Let this solution incubate for 3-3.5 $\mathrm{min}$, at room temperature.

CRITICAL STEP The KOH:NaOCl solution should be added cold for efficiency purposes. If the procedure is done for many samples, which will require usage of this solution for long periods, the solution should be kept in a Styrofoam box containing ice to prevent the solution from warming up.

8 Centrifuge the tubes at $650 \mathrm{~g}$ for $1 \mathrm{~min}$, at room temperature.

9 Use a Pasteur pipette to transfer the pellet from the tube to one of the 15-ml tubes filled with M9 buffer at step 4 above.

CRITICAL STEP The overall duration of steps 7 through 9 needs to be tightly controlled and be kept between 4.5 and $5.5 \mathrm{~min}$. A shorter incubation time could result in adults surviving this process, and thus, synchronization of the offspring developmental time would not be achieved. Longer incubation times could lead to embryo inviability.

10 Centrifuge the tubes from step 9 at $650 \mathrm{~g}$ for $1 \mathrm{~min}$, at room temperature.

11 Discard the supernatant and refill the tube with $10 \mathrm{ml}$ of M9.

12 Repeat steps 10 and 11. 
13 Add 3-5 $\mathrm{ml}$ of $\mathrm{M} 9$ buffer to the tubes and let them incubate for $24+/-2 \mathrm{~h}$ at $20{ }^{\circ} \mathrm{C}$ and $80 \%$ relative humidity, with constant shaking at $120 \mathrm{rpm}$.

CRITICAL STEP The tubes should be tilted to ensure sufficient oxygenation of the M9 buffer.

14 The following day, centrifuge the tubes at low speed (70 g) for $1 \mathrm{~min}$, at room temperature.

15 Use a Pasteur pipette to discard the pellet, which contains mostly dead adults.

16 Estimate the density of live larvae (L1 stage) by counting numbers in three droplets of 5- $\mu 1$ each. Use for this purpose the dissecting stereoscope at a magnification between 20x and 30x.

CRITICAL STEP It is important to keep the density of larvae within reasonable boundaries, both for the estimation procedure and for the amount of liquid that will be pipetted onto the NGM plate. If numbers per drop are below 25 or above 80 , densities should be adjusted either by: removal of M9 buffer after centrifugation at $650 \mathrm{~g}$ for $1 \mathrm{~min}$, at room temperature, in the first case, or by adding more M9 buffer, in the second.

? TROUBLESHOOTING

17 Seed 1,000 L1 larvae into a new NGM plate with bacteria.

18 Repeat steps 3 through 17 twice, to obtain two generations of maintenance in the same environment of the competition assay. Using the last generation cultured before the assay, seed as many plates of the line that will be used as resident population as the number of replicates that will be needed (see relevant discussion in the Introduction to determine the number of experimental replicates). Two plates of the invader line are usually enough.

?TROUBLESHOOTING

\section{Invasion assay}

\section{Timing $20 \mathrm{~d}$ (5 generations)}

$1948 \pm 2 \mathrm{~h}$ after the L1 seeding, in the fourth generation after the initial thawing (step 1), use a worm picker to transfer $n$ individuals (immature late L4s) from the GFP-carrying strain (invaders) into the plates carrying the other strain (resident).

CRITICAL STEP The transfer of invading worms into the competition plates must be made as gently as possible to avoid injuring the worms. Any such injury would inevitably decrease the value of estimated fitness effects, thus introducing a bias. Furthermore, if there is a degree of variability with regard to development stage in the invading line, special care must be taken in order to sample randomly the individuals to be transferred. Moving the plate between picking events and choosing the individual closest to the center of view or any other reference point is a good strategy for sampling. 
20 The following day, resume regular maintenance, as described in steps 4 through 17, for the number of generations intended to reach in the experiment.

\section{Counting GFP-expressing invading individuals}

\section{Timing $5 \mathrm{~min} / \mathrm{plate}$}

21 Count the number of GFP-expressing individuals at the predefined generations. For scoring, use plates right before the $\mathrm{KOH}: \mathrm{NaOCl}$ treatment (starts in step 4). For each plate, take the lid out and, using the fluorescent light and GFP filter in the fluorescent microscope, scan the plate at magnification between $40 \mathrm{x}$ and $60 \mathrm{x}$ to count the number of GFP-expressing (thus fluorescent) individuals.

22 Record the number of GFP-expressing individuals into a table containing information about the plate ID (replicate number) and generation of competition.

23 Resume regular maintenance at step 4.

\section{Invasion simulation and estimation of selection coefficients}

\section{Timing $11 \mathrm{~h}$ (for $10^{7}$ simulation replicates)}

24 Upload the table with the counts of invading individuals observed in the different replicates and over the different generations grown following the invasion.

CRITICAL STEP This table must have already been through quality control filters to ensure that the observed data can be recovered in the simulations, with a reasonable range of parameter values. This can be specially important when large changes frequency changes are observed over subsequent generations, which do not follow any particular trend and may indicate measurement errors in one or more generations. Using only the replicates in which the full sequence of counts (trajectory) is observed in at least a given number of simulations, e.g. 50 simulations in a total of $10^{7}$, provides a sensible way to correct this problem.

25 Identify which trajectories were observed and the number of replicates in which they occurred. From the moment a invading line becomes extinct, its trajectory will be formed by consecutive zero counts.

26 Define parameter set values to match the experimental conditions, such as: population size $(N)$, number of generations $(T)$, initial number of invaders $(n)$; or specific simulation parameters like: number of simulation replicates $(R)$ and selection coefficient $(s)$ of the invading genotype.

27 Run the invasion algorithm as detailed in the following in-text table: 
(i) Choose a selection coefficient $(s)$

(ii) Change the invader frequency $(p)$ according to: $\boldsymbol{p}_{t+1}=\left(\boldsymbol{p}_{t}+\boldsymbol{s} \boldsymbol{p}_{t}\right) / 1+\boldsymbol{s} \boldsymbol{p}_{\mathrm{t}}$, where $\boldsymbol{t}$ is a specific generation

(iii) Simulate drift by sampling a random number of invader genotypes, following a binomial distribution with $p_{t+1}$ as the success probability and $N$ as the sample size

(iv) Record the resulting number of invaders and update $p_{t}$ with the value of $p_{t+1}$

(v) Repeat (ii) through (iv) for the total number of generations $(T)$

(vi) Repeat (ii) through (v) for the total number of simulation replicates $(R)$

28 Count the number of times each observed trajectory was found in the simulations and retrieve each trajectory's probability by dividing this number by $R$, the total number of simulation replicates.

29 Calculate the $\mathrm{Lk}$ estimate of the observed data by summing the logarithm (base 10 or natural) of the trajectory's probabilities.

30 Calculate $\mathrm{Lk}$ values for the different selection coefficients by repeating steps 27-29 with different $s$ values. Values increasing from 0 to 0.5 , with increments of 0.01 , can be used for most cases where the invading line is the fittest one.

31 Calculate the maximum Lk estimate of $s$ and confidence interval after fitting a quadratic curve to the selection coefficients that show the higher Lk values.

?TROUBLESHOOTING

\section{Timing}

Steps 1-3, thaw and growth: $3 \mathrm{~d}$

Steps 4-17, washing, $\mathrm{KOH}: \mathrm{NaOCl}$ treatment and new generation seed: $1 \mathrm{~d}$

Step 18, maintenance for two generations: $8 \mathrm{~d}$

Step 19, invasion setup: 15 min

Step 20, invasion for 5 generations: $20 \mathrm{~d}$

Steps 21-23, counting GFP individuals: $15 \mathrm{~min}$ 
Steps $24-31$, invasion simulation with $10^{7}$ replicates: $11 \mathrm{~h}$

\section{TROUBLESHOOTING}

TABLE 1 Troubleshooting table.

TABLE 1 Troubleshooting table.

\begin{tabular}{|c|c|c|c|}
\hline Step & Problem & Possible reason & Solution \\
\hline \multirow[t]{2}{*}{16} & $\begin{array}{l}\text { Insufficient amount of live L1 } \\
\text { larvae to start next generation }\end{array}$ & $\begin{array}{l}\text { Excessive action of } \\
\mathrm{KOH}: \mathrm{NaOCl} \text { solution in } \\
\text { steps } 7 \text { through } 9\end{array}$ & $\begin{array}{l}\text { Make sure the total time during which } \\
\text { the solution is acting at its highest } \\
\text { concentration does not go beyond } \\
5.5 \text { min and that the volume ratio of this } \\
\text { solution when mixed with M9 in step } 7 \\
\text { is 50:50 }\end{array}$ \\
\hline & & $\begin{array}{l}\text { Insufficient removal of the } \\
\mathrm{KOH}: \mathrm{NaOCl} \text { solution in } \\
\text { steps } 10 \text { through } 12\end{array}$ & $\begin{array}{l}\text { To discard the supernatant completely, } \\
\text { the conical tubes have to be fully turned } \\
\text { over. The pellet is usually not disturbed } \\
\text { by this action }\end{array}$ \\
\hline 16 & $\begin{array}{l}\text { Live individuals at different } \\
\text { developmental stages present } \\
\text { in the M9 before seeding }\end{array}$ & $\begin{array}{l}\text { Individuals were not killed } \\
\text { by the action of the } \\
\mathrm{KOH}: \mathrm{NaOCl} \text { solution }\end{array}$ & $\begin{array}{l}\text { Make sure the } \mathrm{KOH}: \mathrm{NaOCl} \text { solution is } \\
\text { cold when applied and that the volume } \\
\text { ratio of this solution when mixed with } \\
\text { M9 in step } 7 \text { is } 50: 50\end{array}$ \\
\hline 18 & $\begin{array}{l}\text { Not enough L1 larvae } \\
\text { available to seed the intended } \\
\text { number of replicate resident } \\
\text { populations }\end{array}$ & $\begin{array}{l}\text { Problem with } \mathrm{KOH}: \mathrm{NaOCl} \\
\text { procedure in step } 16 \text { or } \\
\text { intrinsically low growth rate } \\
\text { of the resident population }\end{array}$ & $\begin{array}{l}\text { Increase the number of NGM plates that } \\
\text { are seeded before the invasion setup or } \\
\text { increase the number of generations } \\
\text { between thawing and the invasion }\end{array}$ \\
\hline \multirow[t]{3}{*}{31} & $\begin{array}{l}\text { Large confidence interval on } \\
\text { selection coefficients estimates }\end{array}$ & $\begin{array}{l}\text { Lack of statistical power of } \\
\text { the experimental data }\end{array}$ & $\begin{array}{l}\text { Increase the number of invasion } \\
\text { replicates }\end{array}$ \\
\hline & & & $\begin{array}{l}\text { Increase the number of generations of } \\
\text { invasion }\end{array}$ \\
\hline & & & $\begin{array}{l}\text { Increase the number of simulation } \\
\text { replicates }\end{array}$ \\
\hline
\end{tabular}




\section{ANTICIPATED RESULTS}

Expect the observed frequencies of invaders to wander around a low value during the invasion, from which extinctions can occur. Although no absolute empirical thresholds exist, theory predicts that above $\ln (2) / \mathrm{s}$ individuals (s, selection coefficient) the frequency of extinctions should be negligible ${ }^{4}$. In a previous experiments, starting with two invading individuals, extinctions occurred in $37 \%$ of cases (out of a total of 35 replicates) after five generations, even if the invader line had a high selection coefficient ${ }^{8}$. Most of the observed trajectories should be recovered in the simulations, which will result in a bell-shaped curve to be obtained for the likelihood estimates.

Supplementary Table 1, containing data from an experiment starting with two invading individuals is provided where competitions experiments ran for five generations and scoring was obtained for generations 3 through 5 .

\section{SUPPLEMENTARY INFORMATION}

A table with observed counts, "Supplementary Table 1 _Example_counts" is given as supplementary information, together with a script file, "Supplementary Data _ Invasion script" , which gives the implementation in $\mathrm{R}$ code of the invasion simulation and estimation of selection coefficients described in steps 24 through 31. Supplementary Figure 1 is also provided to show the effect of increasing the number of generations in the estimation procedure.

\section{AUTHOR CONTRIBUTIONS STATEMENTS}

I.M.C designed the protocol, wrote the simulation and analysis script and wrote the manuscript.

\section{ACKNOWLEDGMENTS}


I thank Isabel Gordo and Henrique Teotónio for advice in the development and implementation of this protocol. I also thank the reviewers for suggestions and comments, which significantly improved the manuscript. This work was only possible with the support of FCT Investigator program IF/00031/2013 and EXPL/BIA-EVF/1211/2013 to Ivo M. Chelo and financial and technical support from Henrique Teotónio, through the Human Frontiers Science Program (RGP0045/2010) grant.

\section{COMPETING FINANCIAL INTERESTS}

The author declares no competing financial interests.

\section{REFERENCES}

1. Orr, H. A. Fitness and its role in evolutionary genetics. Nat. Rev. Genet. 10, 531-539 (2009).

2. Hartl, D. L. \& Clark, A. G. Principles of Population Genetics. Edn 3. (Sinauer Associates, Sunderland, Massachusetts, USA, 1997).

3. Fisher, R. On the dominance ratio. Proc. Royal Soc. Edinburgh 42, 321-341 (1922).

4. Haldane, J. A mathematical theory of natural and artificial selection, part V: selection and mutation. Math. Proc. Cambridge Phil. Soc. 23, 838-844 (1927).

5. Heffernan, J. M. \& Wahl, L. M. The effects of genetic drift in experimental evolution. Theor. Popul. Biol. 62, 349-356 (2002).

6. Gifford, D. G., de Visser, J. A. \& Wahl, L. M. Model and test in a fungus of the probability that beneficial mutations survive drift. Biol. Lett. 9, 2012.0310 (2012).

7. Roff, D. A. Defining fitness in evolutionary models. J. Genet. 87, 339-348 (2008).

8. Chelo, I. M., Nédli, J., Gordo. I. \& Teotónio, H. An experimental test on the probability of extinction of new genetic variants. Nat. Commun. 4, 2417 (2013).

9. Fisher, R. The genetical theory of natural selection. Clarendon Press, Oxford (1930).

10. Stiernagle T. Maintenance of C. elegans. In C. elegans: a Practical Approach ( ed. Hope, I. A.) (Oxford University Press, Oxford, 1999).

11. Teotónio, H., Carvalho, S., Manoel, R., Roque, M. \& Chelo I. M. Evolution of outcrossing in experimental populations of Caenorhabditis elegans. PLoS One 7 (2012). 
12. Chelo I. M. \& Teotónio, H. The opportunity for balancing selection in experimental populations of Caenorhabditis elegans. Evolution 67, 142-156 (2012).

13. Fire, A. et al. Potent and specific genetic interference by double-stranded RNA in Caenorhabditis elegans. Nature 391, 806-811 (1998).

14. Metz, J. A. J., Nisbet, R. M. \& Geritz, S. A. H. How should we define 'fitness' for general ecological scenarios? Trends. Ecol. Evol. 7, 198-202 (1992).

15. R Development Core Team. R: A language and environment for statistical computing http://www.R-project.org (2006).

16. Elena, S. F. \& Lenski, R. E. Evolution experiments with microorganisms: the dynamics and genetic bases of adaptation. Nat. Rev. Genet. 4, 457-469 (2003).

17. Perfeito, L., Fernandes, L., Mota, C. \& Gordo, I. Adaptive mutations in bacteria: high rate and small effects. Science 317, 813-815 (2007).

18. Wright, S. Evolution in Mendelian populations. Genetics 16, 97-159 (1931).

19. Chevin, L. M. On measuring selection in experimental evolution. Biol. Lett. 7, 210-213 (2011).

20. Doebeli, M. \& Dieckmann, U. Evolutionary branching and sympatric speciation caused by different types of ecological interactions. Am. Nat. 156, S77-S101 (2000).

21. Mylius, S. D. \& Dieckmann, O. The resident strikes back: invader-induced switching of resident attractor. J. Theor. Biol. 211, 297-311 (2001).

22. Yanik, M. F., Rohde, C. B. \& Pardo-Martin, C. Technologies for micromanipulating, imaging and phenotyping small invertebrates and vertebrates. Annu. Rev. Biomed. Eng. 13, 185-217 (2011). 


\section{FIGURE LEGENDS}

Figure 1. Workflow of the protocol to determine invasive fitness. (a) Replicate invasions, starting from low numbers of invaders, are maintained for several generations while scoring takes place. (b) Computer simulations are used to generate possible frequency trajectories under different selection coefficients of the invading genotype. The likelihood curve is then obtained from the frequency at which the observed counts are found in the simulations. G: generation.

Figure 2. Effect of replicate number on fitness estimation. For different simulated sets, generated with $10,30,50$ and 100 replicate invasions and selection coefficients of $0,0.001,0.01$, $0.05,0.1,0.2,0.3$ and 0.5 , maximum likelihood (ML) estimates and $-2 \operatorname{lnLk}$ confidence intervals were obtained to show how replicate number affect the accuracy (a), "statistical" power (see definition below) (b) and precision (c) of the estimation procedure. For each parameter combination, 100 simulations were carried out.

Each invasion started with two individuals in a resident population of 1000 individuals and continued for five generations. Only data from generations 3, 4 and 5 were used. ML estimation is based on $10^{7}$ simulations for each of 81 selection coefficients (from -0.1 to 0.7 in steps of 0.01 ). (a) The mean (symbols) and standard deviation (error bars) of estimated s ( $\hat{\mathrm{s}}$ ) are shown. To avoid complete visual overlap of the estimates from the different replicate numbers, noise was added to the x coordinates (jitter function in R) in this panel. (b) The estimation of "statistical" power is provided by the proportion of times the 'true' selection coefficient is found in the $-2 \operatorname{lnLk}$ confidence interval. With the exception of the lowest replicated data set, power is always higher than 0.9. (c) The size of the -2 lnLk confidence interval (mean and standard deviation are shown) reveals the importance of replicate number in determining the precision with which estimations can be made. 



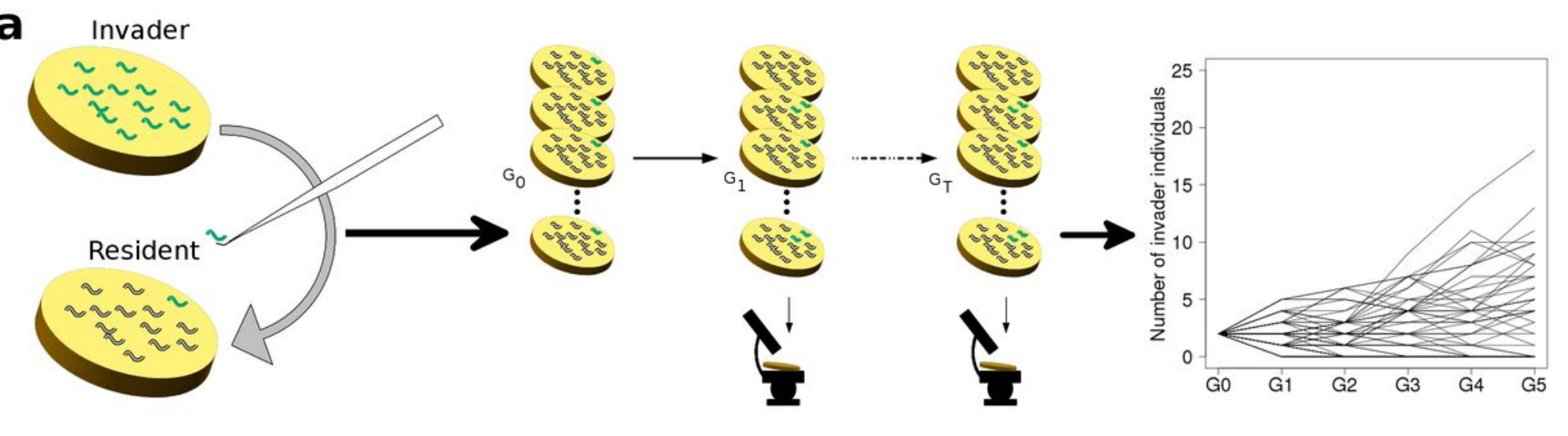

b
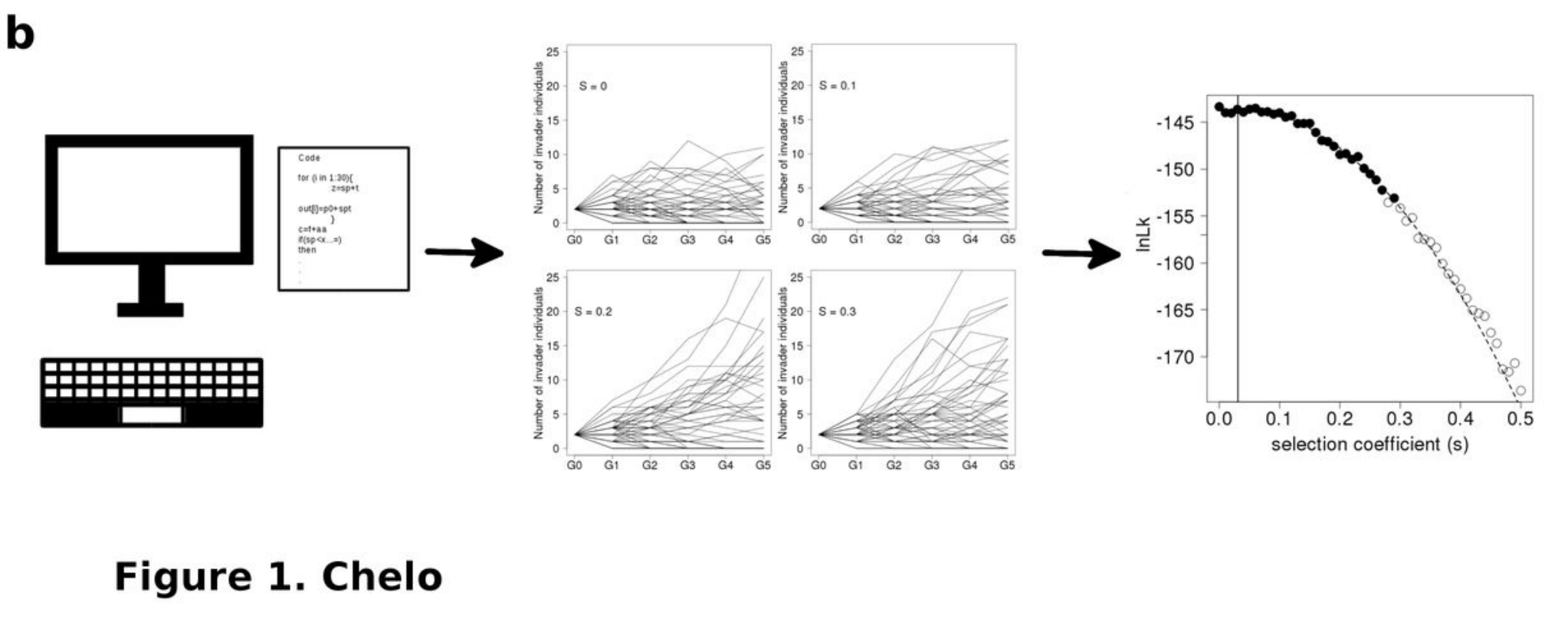

Figure 1. Chelo
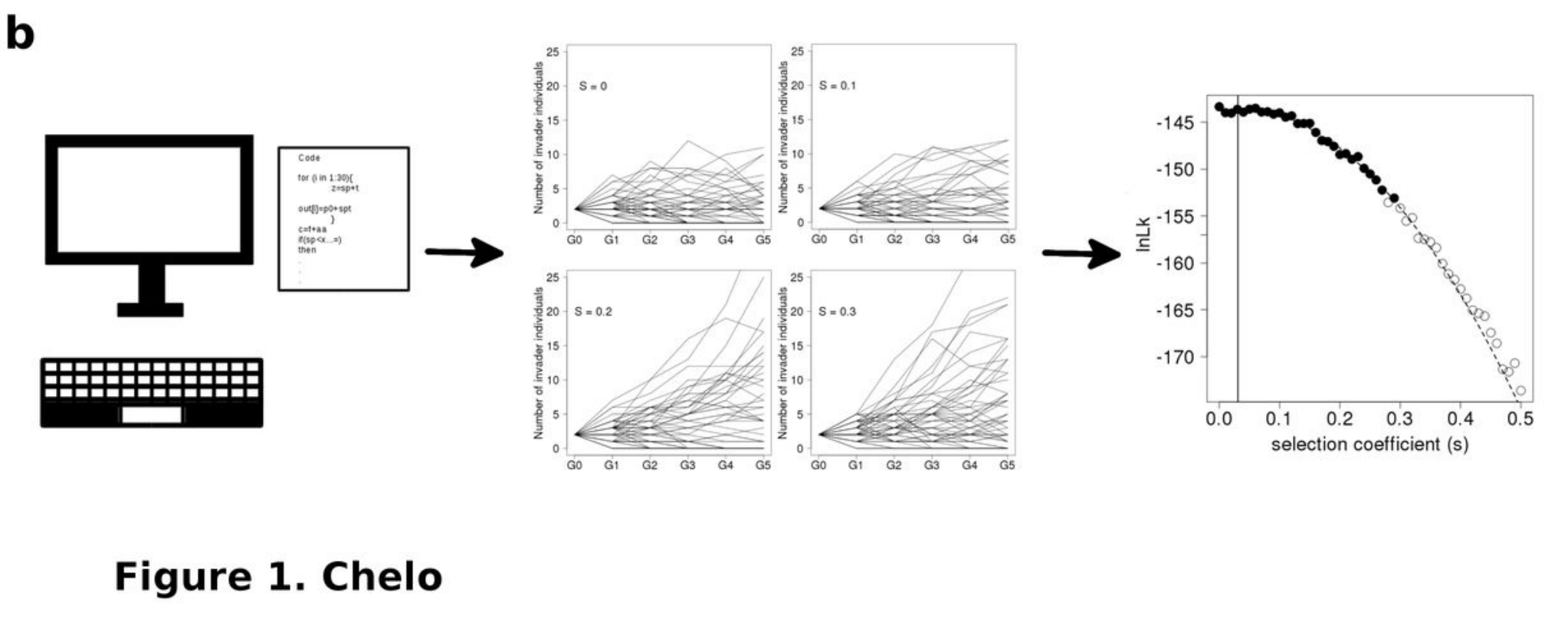

.
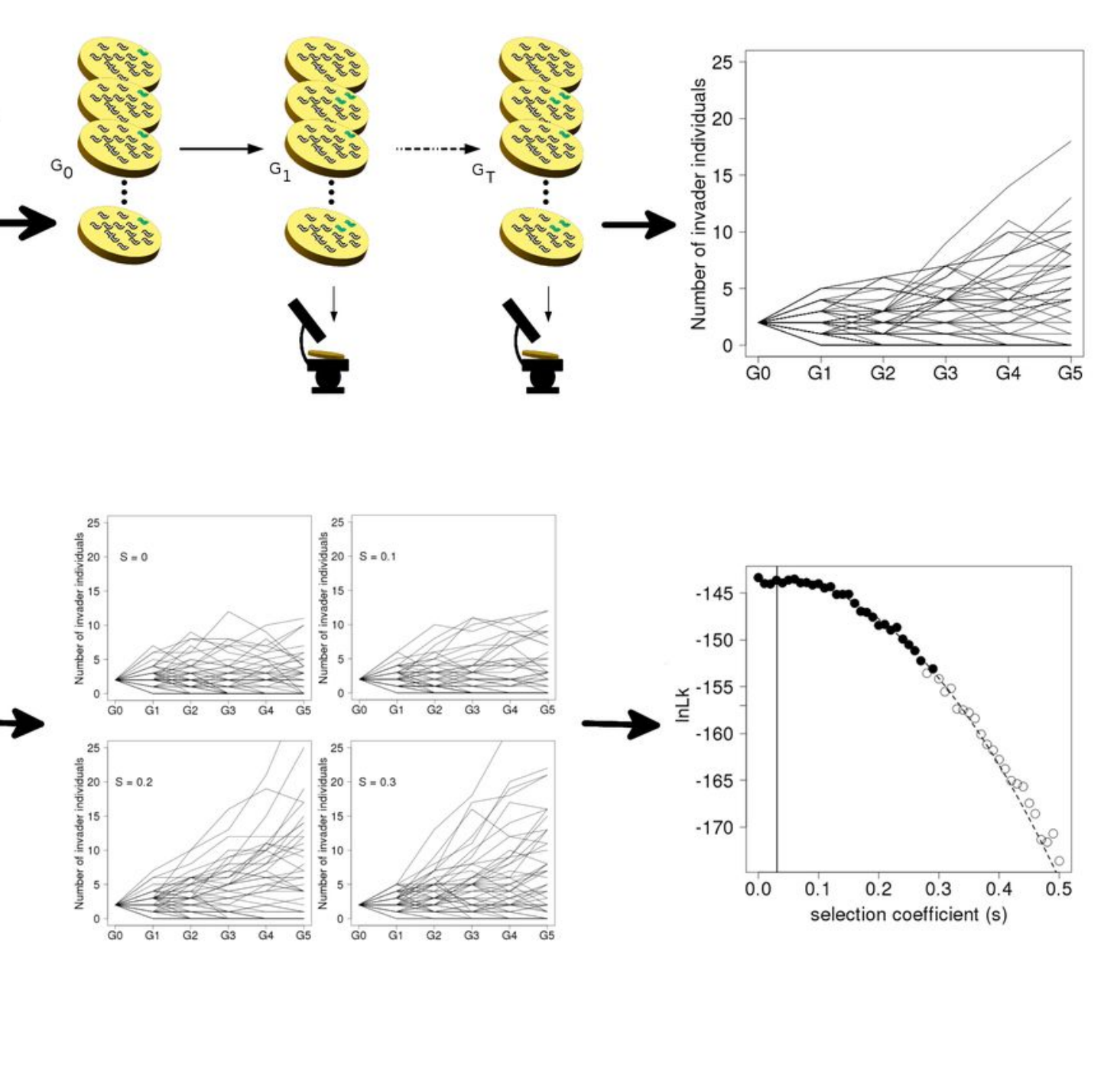

. .

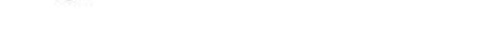

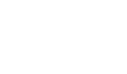

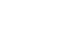



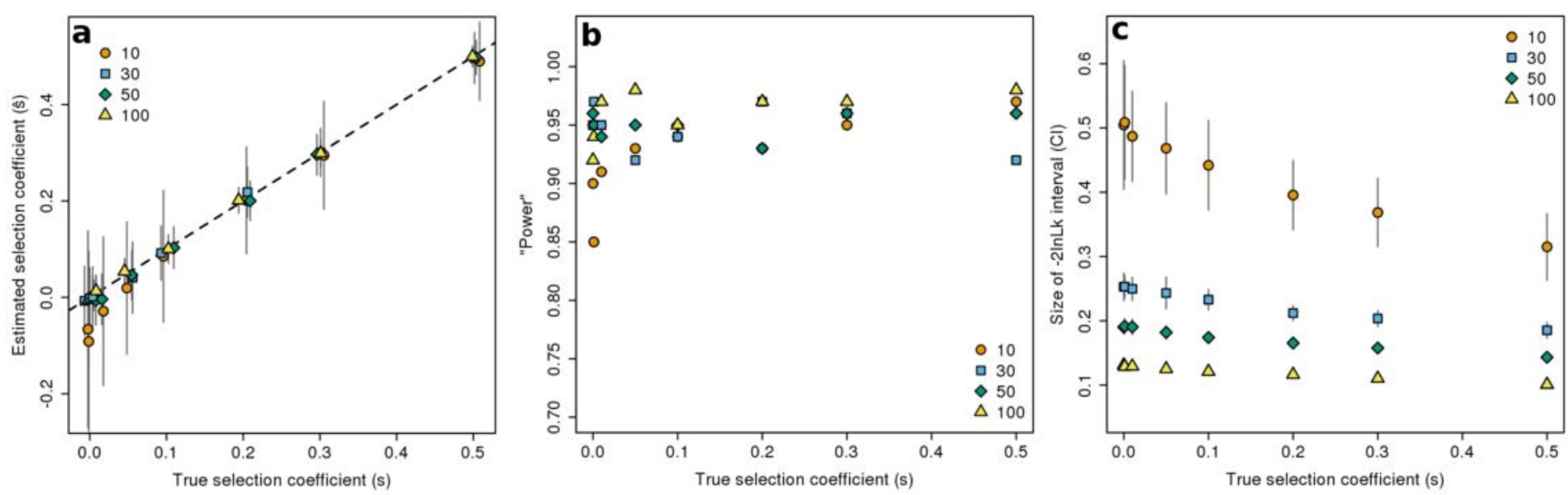

Figure 2. Chelo 

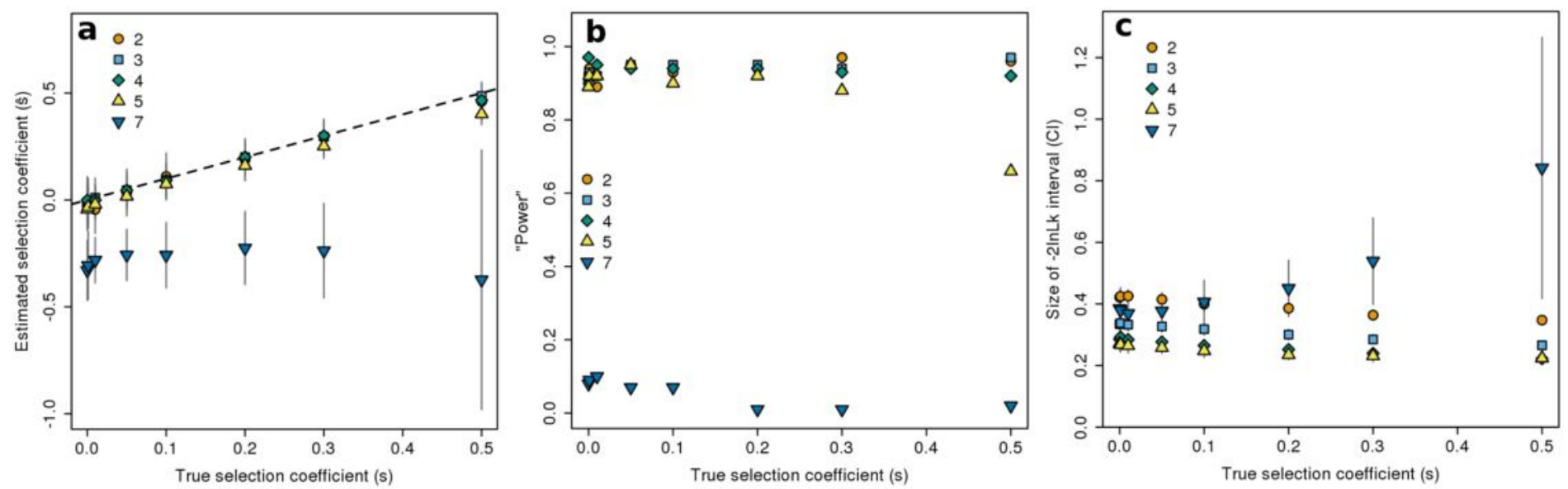

\section{Supplementary Figure 1. (Chelo) Effect of generation number on fitness estimation}

Different sets were simulated for $2,3,4,5$ and 7 generations and selection coefficients of $0,0.001,0.01,0.05,0.1,0.2,0.3$ and $0.5,30$ replicates were used. Maximum likelihood (ML) estimates and - 2 InLk confidence intervals were obtained to show how generation number affects the accuracy (a), "statistical" power (see definition below) (b) and precision (c) of the estimation procedure. For each parameter combination, 100 simulations were carried out. Each invasion started with two individuals in a resident population of 1000 individuals. Data from every generation was used. ML estimation is based on $10^{\wedge} 7$ simulations for each of 61 selection coefficients (from -0.1 to 0.5 in steps of 0.01 ). (a) The mean (symbols) and standard deviation (error bars) of estimated s ( $\hat{s})$ are shown. (b) The estimation of "statistical" power is provided by the proportion of times the 'true' selection coefficient is found in the -2 InLk confidence interval. (c) The size of the -2 InLk confidence interval (mean and standard deviation are shown) reveals how the number of generations affect precision. The increase in number of possible trajectories with the number of generations results in a lower power and downward bias in the estimation of selection coefficients. 